\title{
Phonon-phonon interactions due to non-linear effects in a linear ion trap
}

\author{
Cyrille Marquet $^{1 *}$ Ferdinand Schmidt-Kaler ${ }^{2}$ \\ and Daniel F. V. James ${ }^{1 \dagger}$ \\ 1 Theoretical Division T-4, Los Alamos National Laboratory, \\ Mail Stop B-283, P.O. Box 1663, Los Alamos, NM 87545, USA \\ 2 Institut für Experimentalphysik, Universität Innsbruck, \\ Technikerstraße 25, A-6020 Innsbruck, Austria
}

August 25, 2018

\begin{abstract}
We examine in detail the theory of the intrinsic non-linearities in the dynamics of trapped ions due to the Coulomb interaction. In particular the possibility of mode-mode coupling, which can be a source of decoherence in trapped ion quantum computation, or, alternatively, can be exploited for parametric down-conversion of phonons, is discussed and conditions under which such coupling is possible are derived.
\end{abstract}

PACS numbers: $32.80 . \mathrm{Qk}, 42.50 . \mathrm{Vk}, 89.80 .+\mathrm{h}$

LA-UR-02-6745

To be submitted to Applied Physics B.

\footnotetext{
*Permanent address: MIP, Université Pierre et Marie Curie and Département de Physique, École Normale Supérieure, 75005 Paris, France.

${ }^{\dagger}$ To whom correspondence should be addressed; TEL: +(505)-667-5436, FAX: + (505)667-1931, email: dfvj@lanl.gov
} 


\section{Introduction}

Cold ions confined in electromagnetic traps and cooled by means of lasers are a very important experimental system both for the study of fundamental physics, such as cold non-neutral plasmas or quantum dynamics, and for technological applications such as optical frequency standards. In the past few years systems of this kind have been the subject of intense study as a possible architecture for the realization of a quantum computer $[1,2,3]$ (for reviews of progress towards this goal, see $[4,5,6,7]$ ). In comparison with other experimental systems of investigations of fundamental quantum phenomena, cold-trapped ions offer a number of advantages. In particular, it is a relatively "clean" system whose behavior is well characterized by theory; simplified models of interactions between two-level systems and quantum harmonic oscillators can be realized experimentally. Considerable achievements in the production of non-classical states of matter [8] and other fundamental physics problems such as high-efficiency measurements of Bell's inequalities [9] or tests of cavity quantum-electrodynamics [10] have been made.

As is well known [11], it is impossible to confine charged particles by electrostatic forces alone. To overcome this problem, the radio-frequency Paul trap was developed: such devices create an effective binding potential in the $x$ and $y$ directions while a weaker static potential is applied in the $z$ direction [12]. When two or more ions are confined in such a trap, they will repel each other due to the Coulomb force, resulting in confined charged plasmas with very low densities. When sufficiently cold, the plasma will condense into a crystalline state. In the highly anisotropic traps used for some atomic clocks [13] and for quantum computing, this crystalline state is, for small enough numbers of ions, a simple chain of ions lying in a straight line along the axis of weak binding. In what follows, we shall be examining the oscillatory of ions confined in such a linear configuration.

The ions are assumed to be sufficiently cold that they undergo only small oscillations around their equilibrium positions. In this case, the Lagrangian of the motion can be expanded as a Taylor series around these equilibrium positions. As we shall see, the first term of this expansion is a constant (which has no effect on the motion), the second term vanishes (by virtue of the condition for equilibrium); the third term describes coupling of one ion's motion to all of the others (and can be easily resolved into a series 
of normal modes describing the ions' collective oscillations); the fourth term (whose consequences are the main theme of this paper), describes an intrinsic coupling between the different modes.

The effects of non-linearities in the coupling of harmonically bound particles is a problem with a long history: for example Fermi, Pasta and Ulam investigated such dynamics computationally in 1954-55, using the early Los Alamos MANIAC computer, with a view to explaining the equipartition of energy between modes as the system reaches thermal equilibrium [14]. In the quantum realm, the non-linearities lead to couplings between the normal modes of oscillation. In certain ways this coupling is analogous to $\chi^{(2)}$ non-linear optical effects [15], in that, provided certain conditions are met, quanta of one oscillatory mode can be down-converted into twinned pairs of quanta of other modes. Obviously, since the Taylor expansion is an infinite series, higher terms, describing more complex multi-mode couplings, will be present; we will, however, not consider them in this current work.

The paper is organized as follows. In Section 2 the Lagrangian of the ion motion is presented and the derivation of the normal modes of the ion oscillations is reprised; the classical description of mode-mode coupling in terms of the Hamiltonian, is formulated. In section 3 the properties of the mode-mode coupling coefficients are discussed, and section 4 discusses the quantum theory of this coupling and the population transfers that it can cause. Section 5 summarizes our conclusions.

\section{Lagrangian of the ion motion in the trap}

Consider $N$ identical ions of mass $M$ and charge $Q$ confined in an effective three-dimensional harmonic potential ${ }^{1}$. The position of the $n$-th ion will be denoted $\left(x_{n 1}, x_{n 2}, x_{n 3}\right)$ (Fig.1), where the ions are numbered in order of increasing value of their axial positions $x_{n 3}$, so that $n>m$ implies that $x_{n 3}>x_{m 3}$. Besides the trap potential, each ion experiences a Coulomb interaction with each of the other ions. Thus the total potential energy of

\footnotetext{
${ }^{1}$ In reality, the ions are experiencing a time varying force whose effects can be modeled as an effective harmonic motion plus a high-frequency oscillation called micromotion. Experimental techniques for minimizing this effect are explained in ref.[16]. Here we will assume that the amplitudes of transverse oscillations are sufficiently small that micromotion can be neglected in this paper.
} 
the ions is given by the following expression:

$$
V=\frac{M}{2} \sum_{n=1}^{N} \sum_{i=1}^{3} \omega_{i}^{2} x_{n i}^{2}+\frac{Q^{2}}{8 \pi \epsilon_{0}} \sum_{\substack{n, m=1 \\ m \neq n}}^{N}\left[\sum_{i=1}^{3}\left(x_{n i}-x_{m i}\right)^{2}\right]^{-\frac{1}{2}}
$$

where $\omega_{1}, \omega_{2}$ and $\omega_{3}$ are the angular frequencies of the trap in the three directions. For simplicity, we will assume that the trapping potentials are equal in two transverse directions (1 and 2) and that the trapping potential in the axial direction (3) is much weaker i.e.

$$
\omega_{1}=\omega_{2}=\omega_{3} / \sqrt{\alpha}
$$

where $\alpha \ll 1$ is a dimensionless parameter characterizing the anisotropy of the trap.

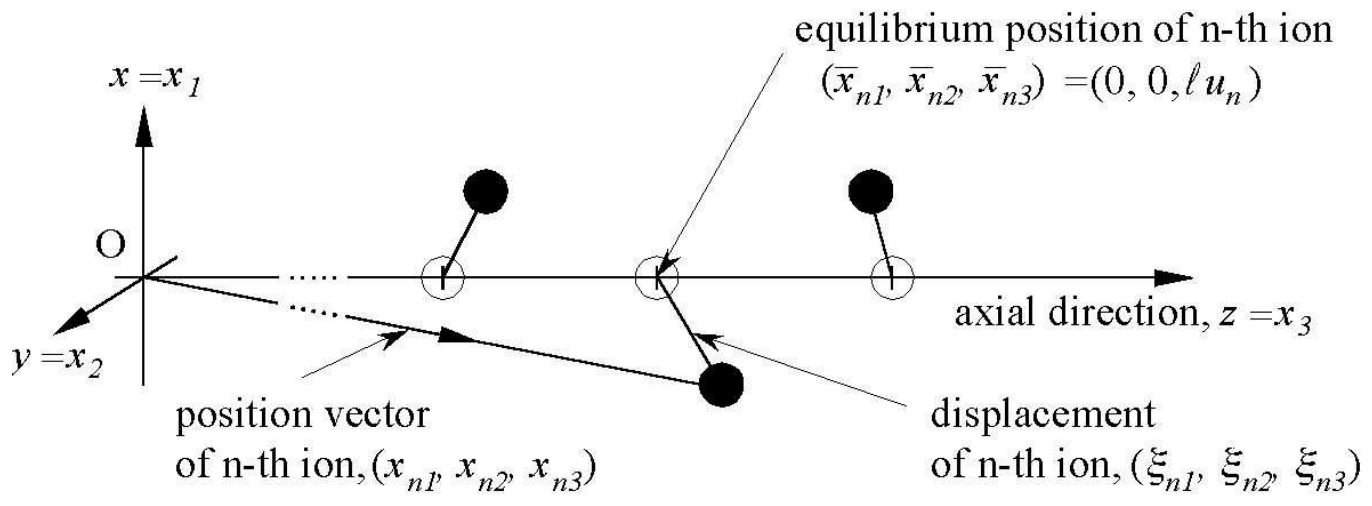

Figure 1: Illustrating the notation used in this paper.

The equilibrium positions of the ions, denoted by $\bar{x}_{n i},(n=1, \ldots, N)$, $(i=1,2,3)$ are determined by the following equations:

$$
\left.\frac{\partial V}{\partial x_{n i}}\right|_{0}=0 \quad(n=1, \ldots, N) \quad(i=1,2,3),
$$

where the subscript 0 denotes that the partial derivatives are evaluated at $x_{n i}=\bar{x}_{n i}$. The solutions of these equilibrium equations in strongly 
anisotropic trapping conditions (so that the ions are aligned along the weak axial direction 3, i.e. $\bar{x}_{n 1}=\bar{x}_{n 2}=0$ ) have been investigated by various authors $[17,18,19]$ (see also [20, 21] for analysis of ion crystals with two different species of ion). It will be convenient to use the dimensionless equilibrium positions defined by $u_{n}=\bar{x}_{n 3} / \ell$ where the length scale $\ell$ is defined by

$$
\ell=\left(\frac{Q^{2}}{4 \pi \epsilon_{0} M \omega_{3}^{2}}\right)^{1 / 3} .
$$

The displacements of the ions from their equilibrium position are denoted $\xi_{n i}$, i.e.

$$
x_{n i}(t)=\bar{x}_{n i}+\xi_{n i}(t) .
$$

The Lagrangian describing the motion is then

$$
\begin{aligned}
L= & T-V \\
= & \frac{M}{2} \sum_{n=1}^{N} \sum_{i=1}^{3} \dot{\xi}_{n i}^{2}-\frac{M}{2} \sum_{n=1}^{N} \sum_{i=1}^{3} \omega_{i}^{2}\left[\bar{x}_{n i}+\xi_{n i}(t)\right]^{2} \\
& -\frac{Q^{2}}{8 \pi \epsilon_{0}} \sum_{\substack{n, m=1 \\
m \neq n}}^{N}\left\{\sum_{i=1}^{3}\left[\bar{x}_{n i}+\xi_{n i}(t)-\bar{x}_{m i}-\xi_{m i}(t)\right]^{2}\right\}^{-\frac{1}{2}} .
\end{aligned}
$$

Making a Taylor expansion about the equilibrium positions, this may be approximated as

$$
\begin{aligned}
L= & \frac{M}{2} \sum_{n=1}^{N} \sum_{i=1}^{3} \dot{\xi}_{n i}^{2}-V_{0}-\left.\frac{1}{2} \sum_{m, n=1}^{N} \sum_{i, j=1}^{3} \frac{\partial^{2} V}{\partial x_{m i} \partial x_{n j}}\right|_{0} \xi_{m i} \xi_{n j} \\
& -\left.\frac{1}{6} \sum_{m, n, p=1}^{N} \sum_{i, j, k=1}^{3} \frac{\partial^{3} V}{\partial x_{m i} \partial x_{n j} \partial x_{p k}}\right|_{0} \xi_{m i} \xi_{n j} \xi_{p k}+\mathrm{O}\left[\xi_{m i}^{4}\right] .
\end{aligned}
$$

Neglecting both the constant $V_{0}$ (which has no effect on the dynamics) and the higher order terms and evaluating the partial derivatives explicitly (see Appendix A), the Lagrangian may be approximated by following expression

$$
\begin{aligned}
L \approx & \frac{M}{2}\left[\sum_{n=1}^{N}\left(\dot{\xi}_{n 3}\right)^{2}-\omega_{3}^{2} \sum_{m, n=1}^{N} A_{m n} \xi_{m 3} \xi_{n 3}\right] \\
& +\frac{M}{2} \sum_{i=1}^{2}\left[\sum_{n=1}^{N}\left(\dot{\xi}_{n i}\right)^{2}-\omega_{3}^{2} \sum_{m, n=1}^{N} B_{m n} \xi_{m i} \xi_{n i}\right] \\
& -\frac{M \omega_{3}^{2}}{2 l} \sum_{m, n, p=1}^{N} C_{m n p} \xi_{p 3}\left(2 \xi_{m 3} \xi_{n 3}-3 \xi_{m 1} \xi_{n 1}-3 \xi_{m 2} \xi_{n 2}\right) .
\end{aligned}
$$


The first term represents the ions oscillations along the axial $(i=3)$ direction, the second oscillations in the transverse directions $(i=1,2)$ and the third term represents coupling between these oscillations which are a direct and unavoidable consequence of the Coulomb interaction between the ions. The tensors $A_{m n}, B_{m n}$ and $C_{m n p}$ are given by

$$
\begin{aligned}
& A_{m n}= \begin{cases}1+2 \sum_{\substack{p=1 \\
p \neq m}}^{N} \frac{1}{\left|u_{m}-u_{p}\right|^{3}} & \text { if } m=n, \\
\frac{-2}{\left|u_{m}-u_{n}\right|^{3}} & \text { if } m \neq n,\end{cases} \\
& B_{m n}=\left(\frac{1}{\alpha}+\frac{1}{2}\right) \delta_{m n}-\frac{1}{2} A_{m n}, \\
& C_{m n p}= \begin{cases}\sum_{\substack{q=1 \\
q \neq m}}^{N} \frac{\operatorname{sgn}(q-m)}{\left(u_{q}-u_{m}\right)^{4}} & \text { if } m=n=p, \\
\frac{-\operatorname{sgn}(p-m)}{\left(u_{p}-u_{m}\right)^{4}} & \text { if } m=n \neq p, \\
0 & \text { if } m \neq n \neq p,\end{cases}
\end{aligned}
$$

where $\operatorname{sgn}(x)$ stands for the sign of $x$ and $\delta_{m n}$ is the Kronecker delta. The third rank tensor $C_{m n p}$ is symmetric under any exchange of two subscripts; thus, for example, $C_{224}=C_{242}=C_{422}=-1 /\left(u_{2}-u_{4}\right)^{4}$ while $C_{442}=C_{244}=$ $C_{424}=1 /\left(u_{2}-u_{4}\right)^{4}$. All the elements of $A_{m n}$ and $C_{m n p}$ can be calculated numerically from the dimensionless equilibrium positions of the ions.

The matrix $A_{m n}$ is real, symmetric and positive definite. Thus its eigenvalues $\mu_{p}$ are non-negative. The eigenvectors $b_{n}^{(p)}(p=1, \ldots, N)$ are defined by the following formula:

$$
\sum_{n=1}^{N} A_{m n} b_{n}^{(p)}=\mu_{p} b_{m}^{(p)} \quad(p=1, \ldots, N),
$$

where the eigenvectors are numbered in order of increasing eigenvalue. The eigenvectors form a complete basis so that

$$
\sum_{p=1}^{N} b_{m}^{(p)} b_{n}^{(p)}=\delta_{m n}, \quad \quad \sum_{m=1}^{N} b_{m}^{(p)} b_{m}^{(q)}=\delta_{p q} .
$$


For any value of $N$, the two first eigenvectors are the center of mass mode $b^{(1)}=1 / \sqrt{N}(1,1, \ldots, 1),\left(\mu_{1}=1\right)$ and the stretch mode $b^{(2)}=(1 / \mathcal{N})\left(u_{1}, u_{2}, \ldots, u_{N}\right)$ $\left(\mu_{2}=3\right)$ where $\mathcal{N}=\sqrt{\sum_{n=1}^{N} u_{n}^{2}}$. All the eigenvalues and eigenvectors can be calculated numerically; their approximate values are given for example in [18] for 2 to 10 ions. The dynamics of these modes have been confirmed directly in experiments [22]. From eq.(2.10) we see that $B_{m n}$ has the same eigenvectors as $A_{m n}$ but different eigenvalues:

$$
\sum_{n=1}^{N} B_{m n} b_{n}^{(p)}=\gamma_{p} b_{m}^{(p)} \quad(p=1, \ldots, N)
$$

where the eigenvalue $\gamma_{p}$ is related to the longitudinal eigenvalue by the formula

$$
\gamma_{p}=\frac{1}{\alpha}+\frac{1}{2}-\frac{\mu_{p}}{2} .
$$

If $\alpha>\alpha_{\text {crit }}=2 /\left(\mu_{N}-1\right)$ the matrix $B_{n m}$ is not positive definite; this implies unstable transverse oscillation modes, and results in the formation of "zig-zag" crystal structures [23]. We shall assume however that $\alpha$ is kept sufficiently small that this situation does not arise. Note that, with this convention, the transverse oscillation modes are numbered in order of decreasing eigenvalue, so that the $p=1$ center of mass mode has the largest eigenvalue.

Using these eigenvectors, the normal modes of the ions' oscillations in the three directions are defined as follows:

$$
\begin{aligned}
X_{p}(t) & =\sum_{n=1}^{N} b_{n}^{(p)} \xi_{n 1}(t), \\
Y_{p}(t) & =\sum_{n=1}^{N} b_{n}^{(p)} \xi_{n 2}(t), \\
Z_{p}(t) & =\sum_{n=1}^{N} b_{n}^{(p)} \xi_{n 3}(t) .
\end{aligned}
$$

The Lagrangian eq.(2.8) can be rewritten in terms of these normal modes quite straightforwardly. Not surprisingly, one finds that, neglecting the fourth order term, the Lagrangian becomes a sum of Lagrangians of harmonic oscillators. The canonical momenta conjugate to $X_{n}$ is $\Pi_{n}^{x}=\partial L / \partial \dot{X}_{n}$ (with analogous definitions for $\Pi_{n}^{y}$ and $\Pi_{n}^{z}$ ). Using these momenta, the Hamiltonian for the ion motion is

$$
H=H_{0}+H_{I},
$$


where $H_{0}$ is the Hamiltonian for all of the uncoupled collective oscillations, i.e.

$$
\begin{aligned}
H_{0} & =\sum_{n=1}^{N}\left[\frac{\Pi_{n}^{x^{2}}}{2 M}+\frac{M \Omega_{n}^{2} X_{n}^{2}}{2}\right] \\
& +\sum_{n=1}^{N}\left[\frac{\Pi_{n}^{y^{2}}}{2 M}+\frac{M \Omega_{n}^{2} Y_{n}^{2}}{2}\right] \\
& +\sum_{n=1}^{N}\left[\frac{\Pi_{n}^{z^{2}}}{2 M}+\frac{M \nu_{n}^{2} Z_{n}^{2}}{2}\right],
\end{aligned}
$$

$\nu_{n}=\omega_{3} \sqrt{\mu_{n}}$ being the angular frequency of the $n$-th mode in the $\mathrm{z}$ direction and $\Omega_{n}=\omega_{3} \sqrt{\gamma_{n}}$ the angular frequency of the $n$-th transverse mode. The Hamiltonian $H_{I}$ describes the perturbation which, in certain circumstances, couples these various modes. It is given by

$$
H_{I}=\frac{M \omega_{z}^{2}}{2 l} \sum_{m, n, p=1}^{N} D_{m n p} Z_{p}\left(2 Z_{m} Z_{n}-3 X_{m} X_{n}-3 Y_{m} Y_{n}\right)
$$

where the mode-mode coupling coefficients $D_{p q r}$ are defined by

$$
D_{p q r}=\sum_{l, m, n=1}^{N} C_{l m n} b_{l}^{(p)} b_{m}^{(q)} b_{n}^{(r)}
$$

Note that the sign of $D_{p q r}$ depends on the sign of the eigenvector $\mathbf{b}^{(p)}$, which is undetermined. We will adopt the convention that the sign of the $N$-th component of $\mathbf{b}^{(p)}$ is always positive, for all modes and values of $N$.

We should mention that other mechanisms by which resonant interactions between ion motion in different directions are coupled have been studied. In particular Werth et al. at the University of Mainz have investigated experimentally the parametric coupling of different stable configurations of molecular ion clouds [24]. Cross-mode couplings due to static field imperfections, which can be a cause of heating or of decoherence, were also discussed in ref.[4] sec 4.1.8.

\section{Properties of the Coupling Coefficients}

In this section we discuss some of the properties of the coefficients $C_{m n p}$ and $D_{m n p}$, which are central to the subsequent development of phonon-phonon 
interactions in ion traps. The first important symmetry property stems directly from the definition of $C_{m n p}$, eq.(2.11): The tensor $C_{m n p}$ is symmetric under exchange of two subscripts; further, because of the definition (2.20), so is $D_{m n p}$. From the definition of $C_{m n p}$ eq.(2.11), we also have the property $C_{m m n}=-C_{n n m}$ (provided that $m \neq n$ ). These properties imply that

$$
\sum_{p=1}^{N} C_{m n p}=0 .
$$

This can be shown as follows: if $m \neq n$, we have $\sum_{p=1}^{N} C_{m n p}=C_{m n n}+$ $C_{m n m}$, since $C_{m n p}$ is always zero if all three indices are different. However, since $C_{m n m}=C_{m m n}=-C_{n n m}=-C_{m n n}$, the sum is zero. If $m=n$, $\sum_{p=1}^{N} C_{m m p}=C_{m m m}+\sum_{p=1, p \neq m}^{N} C_{m m p}$, which, by the definition of $C_{m m m}$, eq.(2.11), must also be zero. The identity eq.(3.1) implies that, since $b^{(1)}=$ $(1 / \sqrt{N})\{1,1, \ldots 1\}$

$$
D_{m n 1} \equiv \frac{1}{\sqrt{N}} \sum_{i, j=1}^{N} b_{i}^{(m)} b_{j}^{(n)} \sum_{k=1}^{N} C_{i j k}=0 .
$$

Thus, when considering the three-mode mixing described by eq.(2.19) the center of mass $(p=1)$ mode has zero coupling strength to any other mode. Physically, this can be explained as follows. The $p=1$ mode is special since it is the only mode in which the center of mass of the crystal is displaced: as can be seen from eq.(2.13), $\sum_{m=1}^{N} b_{m}^{(p)}=0$ if $p \neq 1$. Thus to excite or deexcite the $p=1$ mode requires the application of an external force to change the momentum of the crystal as a whole, while no such force is required to transfer energy between any of the other modes.

This result has important consequences: firstly, if one wanted to avoid mode-mode coupling entirely, for example when performing quantum logic operations between ions, it would behoove one to use the center of mass mode as the "quantum bus" as originally proposed by Cirac and Zoller [1]. However, in some experiments [25], it is found that the quantum state of the center of mass mode is rapidly degraded by excitations from various extraneous sources. Our result eq.(3.2) tends to rule out coupling to other ion oscillation modes which may have been imperfectly cooled as a sources of such heating. Furthermore, when the center of mass mode is heated preferentially, it will not cause heating of the other modes. 
A second interesting property of $C_{m n p}$ can be expressed as follows:

$$
\sum_{p=1}^{N} u_{p} C_{m n p}=\frac{1}{2}\left[\delta_{m n}-A_{m n}\right]
$$

where $A_{m n}$ is the ion coupling tensor defined by eq.(2.9). This result can be demonstrated as follows: if $m \neq n$, then $\sum_{p=1}^{N} C_{m n p} u_{p}=C_{m n n} u_{n}+C_{m n m} u_{m}=$ $\left(u_{n}-u_{m}\right) C_{m n n}=1 /\left|u_{n}-u_{m}\right|^{3}$, where the last step results from the definition of $C_{m n n}$ in terms of the scaled equilibrium positions $u_{p}$. For the case $m=n$, $\sum_{p=1}^{N} C_{m n p} u_{p}=-u_{m} \sum_{p=1, p \neq m}^{N} C_{m m p}+\sum_{p=1, p \neq m}^{N} C_{m m p} u_{p}=-\sum_{p=1, p \neq m}^{N} / \mid u_{p}-$ $\left.u_{m}\right|^{3}$. Comparing these expressions involving the $u_{p}$ 's with the definition of $A_{m n}$, eq.(2.9), we obtain eq.(3.3). This result implies the following

$$
D_{m n 2}=\sum_{i, j=1}^{N} b_{i}^{(m)} b_{j}^{(n)} \sum_{k=1}^{N} \frac{u_{k}}{\mathcal{N}} C_{i j k}=\frac{1-\mu_{m}}{2 \mathcal{N}} \delta_{m n},
$$

where, as before, $\mathcal{N}=\sqrt{\sum_{n=1}^{N} u_{n}^{2}}$. Thus the coupling of the first stretch mode $(p=2)$ is constrained so that it will only be coupled to a single other mode rather than to two distinct modes.

For $N=2$ and $N=3$, we can determine the coefficients $D_{m n p}$ algebraically, using the exact expressions for the equilibrium positions and mode vectors which can be obtained in those two simple cases. We find that

$$
\begin{aligned}
& N=2: D_{222}=-2^{\frac{1}{6}}, \\
& N=3: D_{233}=-\frac{3}{\sqrt{2}}\left(\frac{4}{5}\right)^{\frac{4}{3}}, D_{222}=-\frac{1}{\sqrt{2}}\left(\frac{4}{5}\right)^{\frac{1}{3}} .
\end{aligned}
$$

All the other coefficients must be determined numerically, although this is a reasonably straightforward task. The approximate numerical values of the non-zero coefficients for 2 to 10 ions are given in Tables 2a and 2b (Appendix B).

\section{Quantum motion of the ions}

\subsection{Quantization of the Hamiltonian}

We can now consider the quantum motion of the ions by introducing the following position and momentum operators

$$
Z_{n}(t) \rightarrow \hat{Z}_{n}=\sqrt{\frac{\hbar}{2 M \nu_{n}}}\left(\hat{a}_{n}+\hat{a}_{n}^{\dagger}\right) \quad \Pi_{n}^{z}(t) \rightarrow \hat{\Pi}_{n}^{z}=i \sqrt{\frac{M \hbar \nu_{n}}{2}}\left(\hat{a}_{n}^{\dagger}-\hat{a}_{n}\right),
$$




$$
\begin{aligned}
X_{n}(t) \rightarrow \hat{X}_{n}=\sqrt{\frac{\hbar}{2 M \Omega_{n}}}\left(\hat{b}_{n}+\hat{b}_{n}^{\dagger}\right) & \Pi_{n}^{x}(t) \rightarrow \hat{\Pi}_{n}^{x}=i \sqrt{\frac{M \hbar \Omega_{n}}{2}}\left(\hat{b}_{n}^{\dagger}-\hat{b}_{n}\right), \\
Y_{n}(t) \rightarrow \hat{Y}_{n}=\sqrt{\frac{\hbar}{2 M \Omega_{n}}}\left(\hat{c}_{n}+\hat{c}_{n}^{\dagger}\right) & \Pi_{n}^{y}(t) \rightarrow \hat{\Pi}_{n}^{y}=i \sqrt{\frac{M \hbar \Omega_{n}}{2}}\left(\hat{c}_{n}^{\dagger}-\hat{c}_{n}\right),
\end{aligned}
$$

where, as before, the $Z$-coordinate refers to motion along the axial direction of the trap, while the $X$ - and $Y$-coordinates correspond to displacements in the transverse direction. These operators obey the canonical commutation relations

$$
\begin{gathered}
{\left[\hat{X}_{p}, \hat{\Pi}_{q}^{x}\right]=\left[\hat{Y}_{p}, \hat{\Pi}_{p}^{y}\right]=\left[\hat{Z}_{p}, \hat{\Pi}_{p}^{z}\right]=i \hbar \delta_{p, q}} \\
{\left[\hat{a}_{p}, \hat{a}_{q}^{\dagger}\right]=\left[\hat{b}_{p}, \hat{b}_{q}^{\dagger}\right]=\left[\hat{c}_{p}, \hat{c}_{q}^{\dagger}\right]=\delta_{p, q} .}
\end{gathered}
$$

The Hamiltonian becomes:

$$
\hat{H}=\hat{H}_{0}+\hat{H}_{I}
$$

where

$$
\begin{aligned}
\hat{H}_{0}= & \sum_{n=1}^{N} \hbar\left\{\nu_{n} \hat{a}_{n}^{\dagger} \hat{a}_{n}+\Omega_{n}\left[\hat{b}_{n}^{\dagger} \hat{b}_{n}+\hat{c}_{n}^{\dagger} \hat{c}_{n}\right]\right\} \\
\hat{H}_{I}= & \varepsilon \hbar \omega_{3} \sum_{m, n, p=1}^{N} \frac{D_{m n p}}{\sqrt[4]{\mu_{p}}}\left(\hat{a}_{p}+\hat{a}_{p}^{\dagger}\right)\left(\frac{2}{\sqrt[4]{\mu_{m} \mu_{n}}}\left(\hat{a}_{m}+\hat{a}_{m}^{\dagger}\right)\left(\hat{a}_{n}+\hat{a}_{n}^{\dagger}\right)\right. \\
& \left.-\frac{3}{\sqrt[4]{\gamma_{m} \gamma_{n}}}\left[\left(\hat{b}_{m}+\hat{b}_{m}^{\dagger}\right)\left(\hat{b}_{n}+\hat{b}_{n}^{\dagger}\right)+\left(\hat{c}_{m}+\hat{c}_{m}^{\dagger}\right)\left(\hat{c}_{n}+\hat{c}_{n}^{\dagger}\right)\right]\right)
\end{aligned}
$$

where $\varepsilon$ is a dimensionless quantity which characterizes the strength of the non-linearity; it is given by

$$
\varepsilon=\frac{1}{4 \sqrt{2}}\left[\frac{\hbar \omega_{3}}{\alpha_{\mathrm{fsc}}^{2} M c^{2}}\right]^{1 / 6},
$$

where $\alpha_{\mathrm{fsc}}$ is the fine structure constant. Alternatively, $\varepsilon=\sigma / 4 \ell$, where $\sigma=\sqrt{\hbar / 2 M \omega_{3}}$ is the approximate width of the wavepackets of the individual ions, and $\ell$, defined by eq.(2.4), is the length scale of the order of the ions' separation. divided by the ions' separation. Since both $D_{m n p}$ and $\mu_{p}$ are, at most, of the order of unity, while $\gamma_{p}$ is assumed to be much larger than unity, we have:

$$
\left|\frac{\hat{H}_{I}}{\hat{H}_{0}}\right| \sim \varepsilon .
$$


Values of $\varepsilon$ for a variety of ions and traps are given in Table 1. These show this parameter tends to have a small value in experimental circumstances, implying that the treatment of $\hat{H}_{I}$ as a perturbation can be justified.

\begin{tabular}{|l|l|l|l|}
\hline Ion & $\omega_{3}$ & $\varepsilon$ & $\varepsilon \omega_{3}$ \\
\hline \hline${ }^{9} \mathrm{Be}^{+}$ & $(2 \pi) 5.0 \mathrm{MHz}$ & $1.06 \times 10^{-3}$ & $(2 \pi) 5.30 \mathrm{kHz}$ \\
${ }^{40} \mathrm{Ca}^{+}$ & $(2 \pi) 2.0 \mathrm{MHz}$ & $7.09 \times 10^{-4}$ & $(2 \pi) 1.42 \mathrm{kHz}$ \\
${ }^{88} \mathrm{Sr}^{+}$ & $(2 \pi) 200 \mathrm{kHz}$ & $4.24 \times 10^{-4}$ & $(2 \pi) 85 \mathrm{~Hz}$ \\
${ }^{112} \mathrm{Cd}^{+}$ & $(2 \pi) 2.8 \mathrm{MHz}$ & $6.32 \times 10^{-4}$ & $(2 \pi) 1.77 \mathrm{kHz}$ \\
\hline
\end{tabular}

Table 1: Values of the parameter $\varepsilon$ for various ions and trapping frequencies.

We will denote the eigenstates and eigenvalues of $\hat{H}_{0}$ as:

$$
\begin{aligned}
|\Psi\rangle & =\left|\left\{n_{1}^{x}, \ldots, n_{N}^{x}\right\},\left\{n_{1}^{y}, \ldots, n_{N}^{y}\right\},\left\{n_{1}^{z}, \ldots, n_{N}^{z}\right\}\right\rangle \\
E_{\Psi} & =\hbar \sum_{p=1}^{N} \Omega_{p}\left(n_{p}^{x}+n_{p}^{y}\right)+\nu_{p} n_{p}^{z} .
\end{aligned}
$$

\subsection{Resonance conditions}

Fermi's golden rule [26] implies that population transfer between two eigenstates $|i\rangle$ and $|f\rangle$ of $\hat{H}_{0}$ induced by the interaction $\hat{H}_{I}$ can only occur if the matrix element $\left\langle f\left|\hat{H}_{I}\right| i\right\rangle$ is non-zero and if the energies of $|i\rangle$ and $|f\rangle$ are equal 2 . These conditions allow us to neglect a considerable number of the terms that occur when eq.(4.4) is expanded. For example, because the square roots of the eigenvalues of $A_{m, n}$ are all irrational numbers (with the sole exception of $\left.\mu_{1}\right)$, it follows that $\pm\left(\sqrt{\mu_{p}} \pm \sqrt{\mu_{m}} \pm \sqrt{\mu_{n}}\right) \neq 0$; hence we can neglect all the terms of the form $\left(\hat{a}_{p}+\hat{a}_{p}^{\dagger}\right)\left(\hat{a}_{m}+\hat{a}_{m}^{\dagger}\right)\left(\hat{a}_{n}+\hat{a}_{n}^{\dagger}\right)$. Similarly terms involving three creation or three annihilation operators can be ignored. Making these approximations, and using the symmetry properties of $D_{m n p}$ and the commutation relations (4.2), we obtain the following simplified expression for the interaction Hamiltonian, (in the interaction picture)

$$
\hat{H}_{I} \approx-3 \varepsilon \hbar \omega_{3} \sum_{m, n, p=1}^{N} \frac{D_{m n p}}{\sqrt[4]{\gamma_{m} \gamma_{n} \mu_{p}}}\left[2 \hat{a}_{p}\left(\hat{b}_{m}^{\dagger} \hat{b}_{n}+\hat{c}_{m}^{\dagger} \hat{c}_{n}\right) e^{i \Delta_{m n p}^{(-)} \omega_{z} t}\right.
$$

\footnotetext{
${ }^{2}$ This is equivalent to the energy matching condition in parametric down-conversion. Since here the modes do not represent travelling waves, there is no momentum conservation (or phase matching) condition as there is the non-linear optics.
} 


$$
\left.+\hat{a}_{p}\left(\hat{b}_{m}^{\dagger} \hat{b}_{n}^{\dagger}+\hat{c}_{m}^{\dagger} \hat{c}_{n}^{\dagger}\right) e^{i \Delta_{m n p}^{(+)} \omega_{z} t}\right]+h . a .
$$

where h.a. stands for the Hermitian adjoint of the preceding term, and $\Delta_{m n p}^{( \pm)}=\sqrt{\gamma_{m}} \pm \sqrt{\gamma_{n}}-\sqrt{\mu_{p}}$. We shall refer to the term proportional to $\exp \left(i \Delta_{m n p}^{(-)} \omega_{z} t\right)$, which involves the creation of a transverse phonon and simultaneous annihilation of both a longitudinal and a transverse phonon (and the reverse process, contained within the hermitin adjoint part of eq.(4.9)), as a resonance of the first kind; the term with $\exp \left(i \Delta_{m n p}^{(+)} \omega_{z} t\right)$, (which creates two transverse phonons in different modes by annihilating a longitudinal phonon) will be called a resonance of the second kind.

Since the only experimentally controllable parameter in the definitions of $\Delta_{m n p}^{( \pm)}$is the anisotropy parameter $\alpha$, it is natural to ask at what values of $\alpha$ a resonance can occur. A necessary condition for resonance (i.e. for $\Delta_{m n p}^{(+)}=0$ or $\left.\Delta_{m n p}^{(-)}=0\right)$ is as follows:

$$
\alpha=\frac{16 \mu_{p}}{4 \mu_{p}^{2}+\mu_{m}^{2}+\mu_{n}^{2}-8 \mu_{p}+4 \mu_{p} \mu_{m}+4 \mu_{p} \mu_{n}-2 \mu_{m} \mu_{n}} .
$$

Using the known values of the eigenavalues $\mu_{p}$ (ref.[18], table 2), appropriate values for $\alpha$ can be found straightforwardly. However, not all of these values of $\alpha$ given by (4.10) correspond to a resonance, and one must determine whether or not the appropriate condition for either type of resonance is satisfied by direct substitution into the formulas for $\Delta_{m n p}^{( \pm)}$. Since $\sqrt{\mu_{p}} \neq 0$, resonances of the first kind are only possible when two distinct transverse modes are involved (i.e. $m \neq n$ ); resonances of the second kind can occur involving a single transverse mode (i.e. $m$ can be equal to $n$ ). Resonances of the first kind tend to be very weak; the coupling coefficients do not exceed $1.6 \times 10^{-3}$ for $L \leq 10$, and are always zero for $L \leq 6$. The values of $\alpha$ for the two types of resonance are given in Tables $2 \mathrm{a}$ and $2 \mathrm{~b}$ (Appendix B) and are plotted for different numbers of ions in figure 2. Only those values less than the critical value $\alpha_{\text {crit }}$ are included, since our analysis is based on the assumption that the ions are in a linear configuration.

One important result stems from this analysis. Resonance cannot occur 


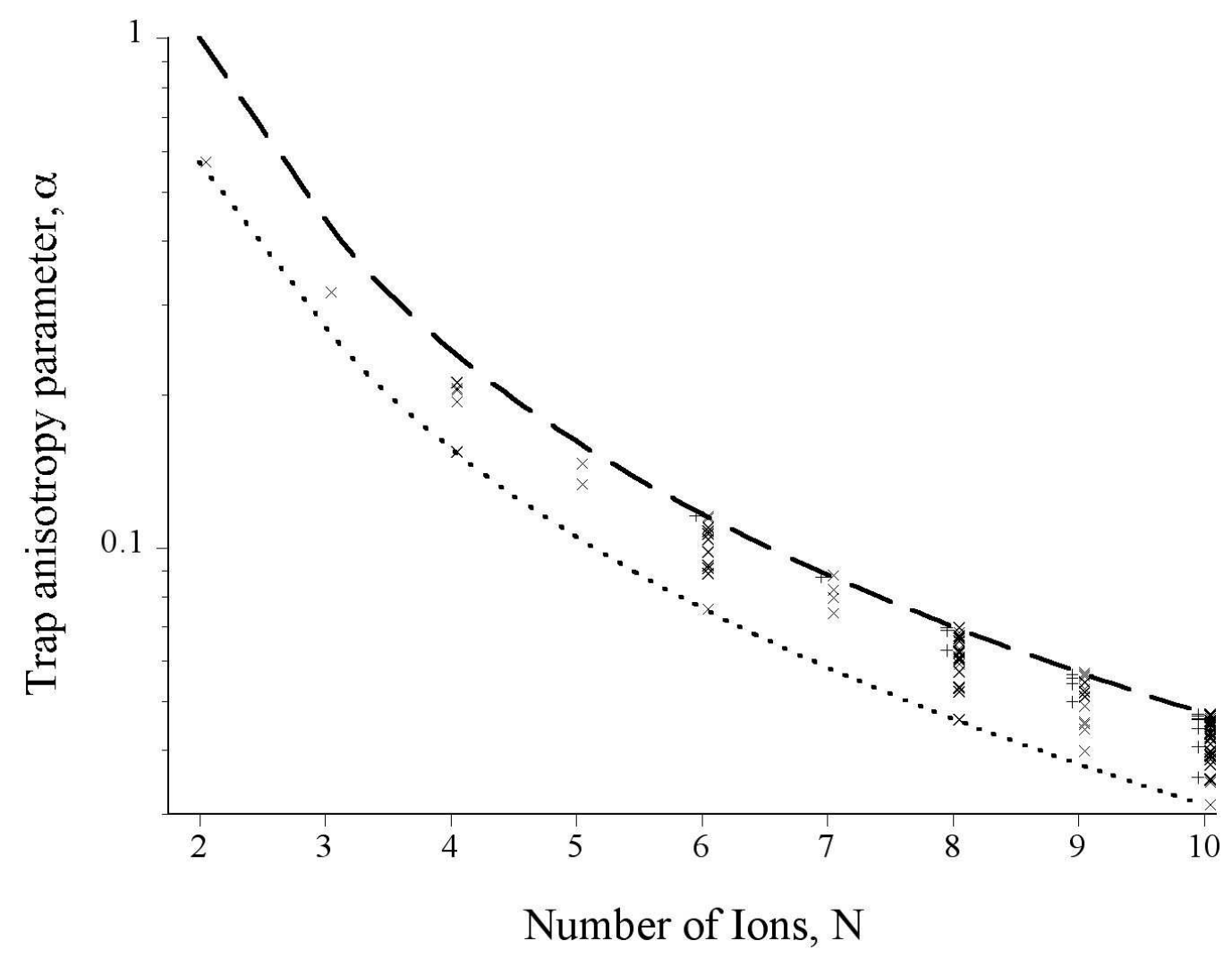

Figure 2: Values of the anisotropy parameter $\alpha$ for which resonant threemode mixing can occur. The horizontal/verticle crosses represent resonances of the first kind $\left(\Delta_{m n p}^{(-)}=0\right)$ and the diagonal crosses represent resonances of the second kind $\left(\Delta_{m n p}^{(+)}=0\right)$. The upper limiting curve represents the critical value $\alpha_{\text {crit }}$, above which the linear confirguration of the ions becomes unstable. The lower curve represents the highest value of $\alpha$ for which resonant mode-mode coupling cannot occur (see eq.(4.11)).

for values of $\alpha$ below the minimum value given by

$$
\alpha_{\text {min }}= \begin{cases}\frac{16 \mu_{N}}{\mu_{N}\left(9 \mu_{N}+2 \mu_{N-1}-8\right)+\mu_{N-1}^{2}}>\frac{4}{3 \mu_{N}-2} & \text { if } N \text { is odd, } \\ \frac{4}{3 \mu_{N}-2} & \text { if } N \text { is even. }\end{cases}
$$

Thus, should one wish to avoid any form of mode-mode coupling due to the forth order terms we are considering in this paper, this can be ensured by using a trap with an anisotropy parameter smaller than $4 /\left(3 \mu_{N}-2\right)$. This can also be expressed in terms of the critical anisoptry for onset of zig-zag, 
$\alpha_{\text {crit }}$ : there will be no three-mode mixing if $\alpha<4 \alpha_{\text {crit }} /\left(\alpha_{\text {crit }}+6\right)$.

\subsection{Example of mode-mode coupling}

As a specific example of the above general analysis, let us assume that we have six ions confined in a trap with anisotropy factor $\alpha=0.09151$. For this value of $\alpha$, a resonance of the second kind $\left(\Delta_{m n p}^{(+)}=0\right)$ occurs for the three modes $(m, n, p)=(5,6,5)$ and also for $(m, n, p)=(6,5,5)$ (since there is symmetry between $m$ and $n$ for resonances of the second kind).

The coupling Hamiltonian can be simplified by neglecting all off-resonant terms; for this case we get

$$
\begin{aligned}
\hat{H}_{I} \simeq & -3 \varepsilon \hbar \omega_{3} \frac{D_{565}}{\sqrt[4]{\mu_{5} \gamma_{5} \gamma_{6}}} \hat{a}_{5}\left(\hat{b}_{5}^{\dagger} \hat{b}_{6}^{\dagger}+\hat{c}_{5}^{\dagger} \hat{c}_{6}^{\dagger}\right) \\
& +-3 \varepsilon \hbar \omega_{3} \frac{D_{655}}{\sqrt[4]{\mu_{5} \gamma_{6} \gamma_{5}}} \hat{a}_{5}\left(\hat{b}_{6}^{\dagger} \hat{b}_{5}^{\dagger}+\hat{c}_{6}^{\dagger} \hat{c}_{5}^{\dagger}\right)+h . a . \\
= & -\varepsilon \hbar \omega_{3} \frac{6 D_{565}}{\sqrt[4]{\mu_{5} \gamma_{5} \gamma_{6}}} \hat{a}_{5}\left(\hat{b}_{5}^{\dagger} \hat{b}_{6}^{\dagger}+\hat{c}_{5}^{\dagger} \hat{c}_{6}^{\dagger}\right)+h . a .,
\end{aligned}
$$

where we have used the fact that $D_{655}=D_{565}$ and that $\left[\hat{b}_{5}^{\dagger}, \hat{b}_{6}^{\dagger}\right]=0$ (and similarly for the $\hat{c}_{m}^{\dagger}$ ). From Table 2 we see that $D_{655}=4.2528$ and $\alpha=0.09151$; from Table 2 of ref.[18] we find $\mu_{5}=13.51$; using eq.(2.15) we find $\gamma_{5}=4.6709$ and $\gamma_{6}=2.2949 ;$ thus $6 D_{655} / \sqrt[4]{\mu_{5} \gamma_{5} \gamma_{6}}=7.3556$.

As an example we will consider the case of coupling between three states defined by

$$
\begin{aligned}
& |\Psi\rangle=|\{0,0,0,0,0,0\},\{0,0,0,0,0,0\},\{0,0,0,0,1,0\}\rangle, \\
& |\Phi\rangle=|\{0,0,0,0,0,0\},\{0,0,0,0,1,1\},\{0,0,0,0,0,0\}\rangle, \\
& |\chi\rangle=|\{0,0,0,0,1,1\},\{0,0,0,0,0,0\},\{0,0,0,0,0,0\}\rangle,
\end{aligned}
$$

where we have used the notation for the motional quantum states of the ion modes defined in eq.(4.7). Note that the coupling defined by eq.(4.12) only involves the 5th longitudinal mode and the 5th and 6th transverse modes; the states of the other modes are unaffected; thus although we have set these other modes to have zero population in the definitions of $|\psi\rangle,|\phi\rangle$ and $|\chi\rangle$, their state can be arbitrary without changing the analysis. The interaction picture state describing the system is given by:

$$
|\Psi(t)\rangle=\psi(t)|\Psi\rangle+\phi(t)|\Phi\rangle+\chi(t)|\chi\rangle
$$


Since, due to the resonance condition, $\left[\hat{H}_{0}, \hat{H}_{I}\right]=0$ and the interaction picture coupling Hamiltonian is:

$$
e^{\frac{i}{\hbar} \hat{H}_{0} t} \hat{H}_{I} e^{-\frac{i}{\hbar} \hat{H}_{0} t}=\hat{H}_{I}
$$

Thus the Schrödinger equation describing the evolution of the system is given by

$$
i \hbar \frac{d}{d t}\left|\Psi_{I}(t)\right\rangle=\hat{H}_{I}\left|\Psi_{I}(t)\right\rangle
$$

which implies that the probability amplitudes obey the following set of coupled equations:

$$
\begin{aligned}
i \dot{\psi}(t) & =-\Gamma[\phi(t)+\chi(t)], \\
i \dot{\phi}(t) & =-\Gamma \psi(t), \\
i \dot{\chi}(t) & =-\Gamma \psi(t),
\end{aligned}
$$

where $\Gamma=\varepsilon \omega_{3}\left(6 D_{565} / \sqrt[4]{\mu_{5} \gamma_{5} \gamma_{6}}\right)$. The solution of these equations can be obtained straightforwardly using Laplace transforms:

$$
\begin{aligned}
\psi(t) & =\psi(0) \cos (\sqrt{2} \Gamma t)+\frac{i(\phi(0)+\chi(0))}{\sqrt{2}} \sin (\sqrt{2} \Gamma t) \\
\phi(t) & =\frac{i \psi(0)}{\sqrt{2}} \sin (\sqrt{2} \Gamma t)+\phi(0) \cos ^{2}\left(\frac{\Gamma t}{\sqrt{2}}\right)-\chi(0) \sin ^{2}\left(\frac{\Gamma t}{\sqrt{2}}\right),(4) \\
\chi(t) & =\frac{i \psi(0)}{\sqrt{2}} \sin (\sqrt{2} \Gamma t)-\phi(0) \sin ^{2}\left(\frac{\Gamma t}{\sqrt{2}}\right)+\chi(0) \cos ^{2}\left(\frac{\Gamma t}{\sqrt{2}}\right) .
\end{aligned}
$$

These solutions are plotted in Figure 3 for the case $\psi(0)=1$ (i.e. the longitudinal phonon mode initially excited) and in Figure 4 for the case $\phi(0)=1$ (i.e. two transverse modes in the $\mathrm{x}$-direction initially excited). 


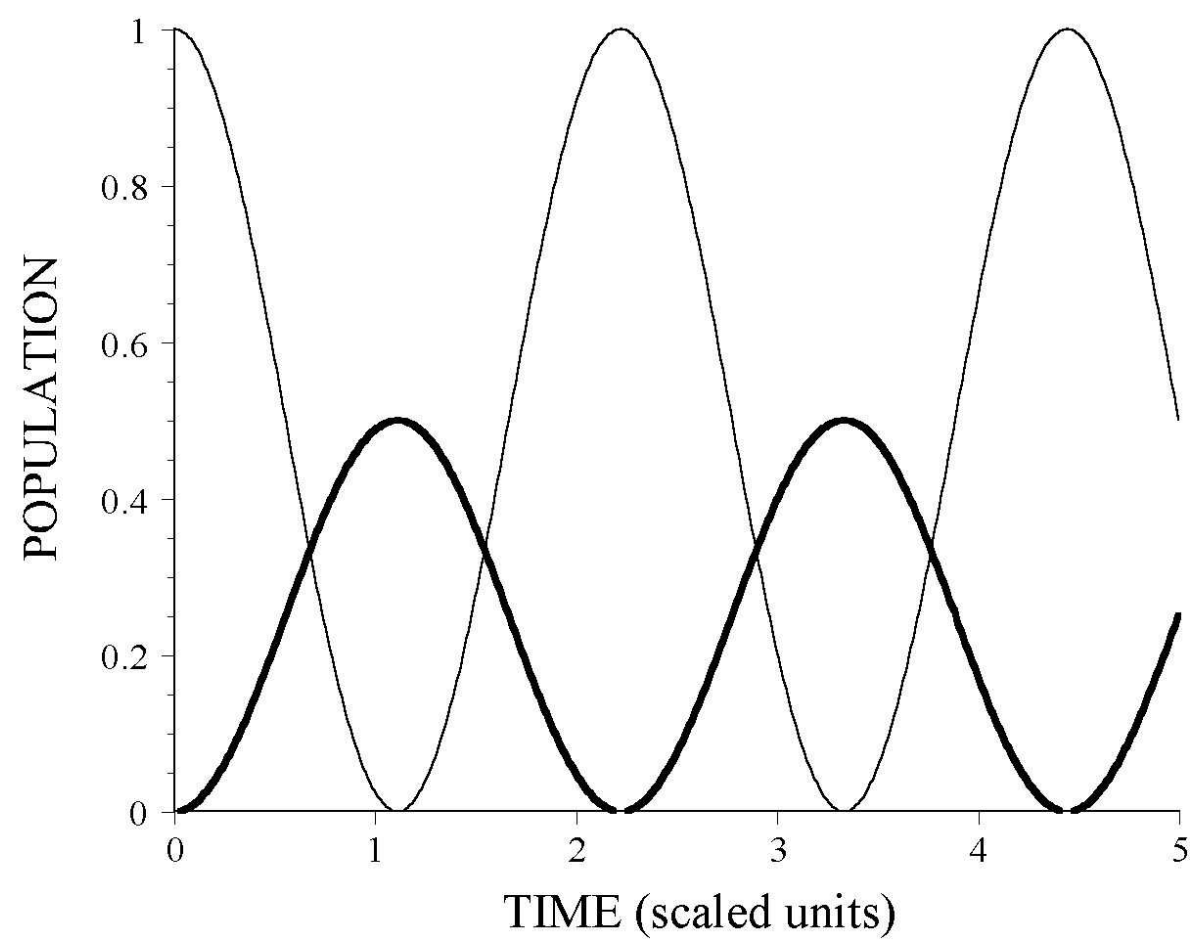

Figure 3: The evolution of the populations when the initial state is $|\psi\rangle$. The thin line represents $|\psi(t)|^{2}$ and the wide line $|\phi(t)|^{2}=|\chi(t)|^{2}$. Time is in units of $1 / \Gamma$.

\subsection{Entanglement}

Let us consider in detail the first example, shown in Fig.3. The crystal of six ions was prepared with a single quanta in the fifth oscillatory mode, i.e. the state

$$
|\Psi(0)\rangle=|\{0,0,0,0,0,0\},\{0,0,0,0,0,0\},\{0,0,0,0,1,0\}\rangle .
$$

After a time $t_{1}=\pi / 2 \sqrt{2} \Gamma$ the crystal will have evolved into the state

$$
\begin{aligned}
\left|\Psi\left(t_{1}\right)\right\rangle= & \frac{i}{\sqrt{2}}(|\{0,0,0,0,0,0\},\{0,0,0,0,1,1\},\{0,0,0,0,0,0\}\rangle \\
& +|\{0,0,0,0,1,1\},\{0,0,0,0,0,0\},\{0,0,0,0,0,0\}\rangle) .
\end{aligned}
$$

This later state has the property of being entangled: it cannot be written as a product of the state of 5 -th and of the 6 -th oscillatory modes. This can be thought of as analogous to the type of polarization entangled states of photon pairs generated in optical down-conversion experiments [27]. The two 


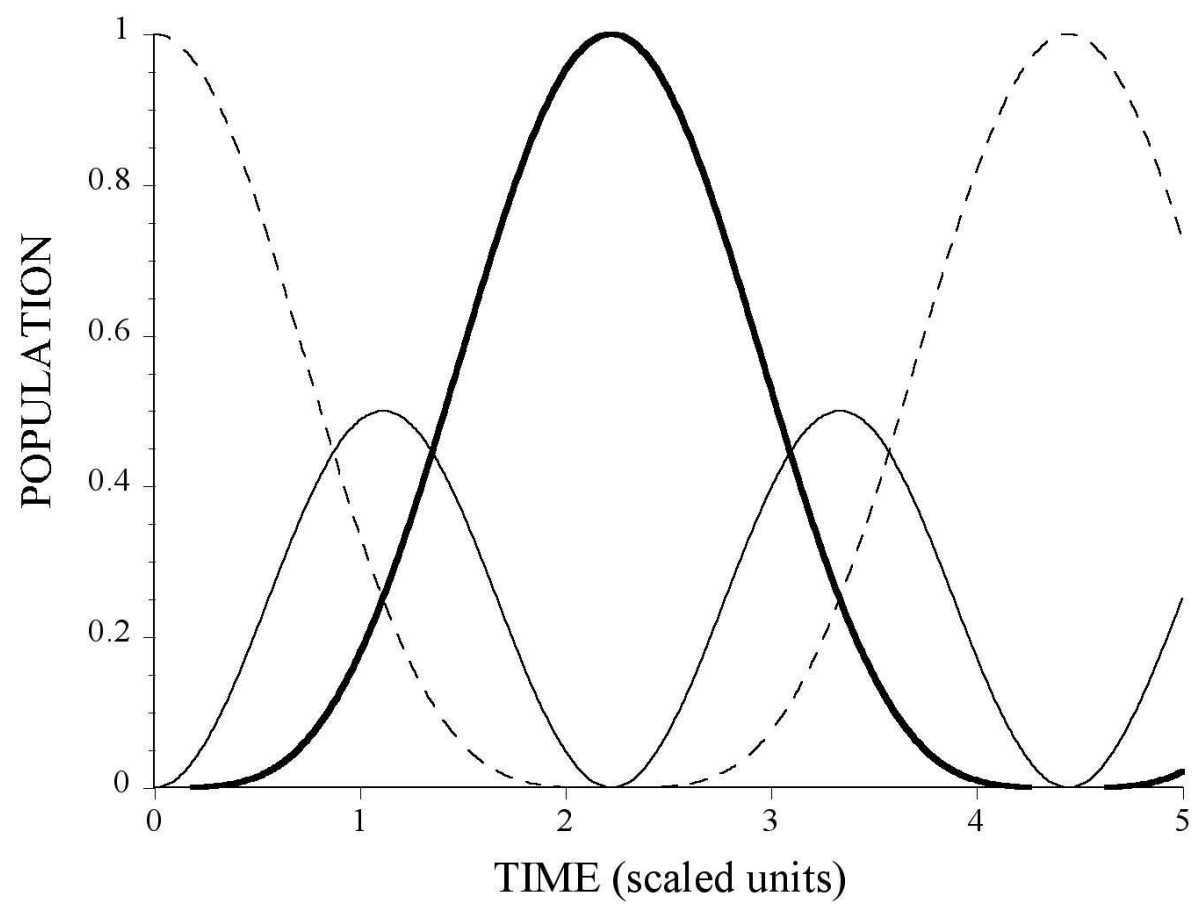

Figure 4: The evolution of the populations when the initial state is $|\phi\rangle$. The thin line represents $|\psi(t)|^{2}$, the dashed line $|\phi(t)|^{2}$ and the wide line $|\chi(t)|^{2}$. Time is in units of $1 / \Gamma$.

transverse directions of oscillation are analogous to the photon polarizations, while the oscillatory mode is analogous to the spatial mode of the photons. The initially excited longitudinal oscillations play the role of the pump laser. Controlling this process in ion traps will be achieved by switching the trap anisotropy parameter $\alpha$ into the appropriate resonance parameter for the appropriate amount of time, then rapidly switching it to a non-resonant value: this will have the effect of turning on the phonon-phonon interaction for a specific time. Furthermore, the technology of preparing oscillatory quantum states of trapped ions is at the moment more advanced than current state of the art for preparing quantum states of the electromagnetic field. Specifically, one can hope to prepare the "pump" longitudinal mode in a single excitation Fock state, while creating single photons is still somewhat problematic. Thus in principle, deterministic preparation of oscillatory entangled states may be attained, in contrast to the non-deterministic, post-selection state preparation in quantum optics experiments. Furthermore the method 
of entangled oscillatory state preparation described here, combined with the variety of techniques for creating entanglement of the internal degrees of freedom raises the possibility of creating hyper-entangled states in ion traps [28].

\section{Conclusion}

In this paper we have analyzed the dynamics of ions confined in harmonic traps. The normal modes of the ions' oscillations are intrinsically coupled by the Coulomb interaction. This "three-mode mixing" is in some ways analogous to non-linear optical effects. There are two important results which our analysis has revealed: first, if one wishes to avoid these effects altogether, the trap anisotropy, characterized by the parameter $\alpha$ defined by eq.(2.2), must be less than a certain value given by eq.(4.11). Secondly, this effect in principle may be exploited to create entangled motional states of the ions, by carefully controlling the ions' dynamics, as outline in section 4.3 and 4.4. How the resulting entangled states may be exploited for tasks in quantum information is a promising avenue for further investigation

\section{Acknowledgments}

The authors would like to thank Dana Berkeland and Eddy Timmermans for useful discussions, Sara Schneider and John Grondalski for reading and commenting on the manuscript and Albert Petschek and William Beyer for drawing our attention to reference [14]. CM would also like to thank Los Alamos National Laboratory for its hospitality during his visit and ENS for providing travel funds. This work was supported in part by the Los Alamos National Laboratory LDRD program.

\section{Appendix A: Derivation of Eqs.(2.9), (2.10) and (2.11).}

In this Appendix, we show how to derive the Lagrangian eq.(2.8) from the eq.(2.7). We will use the following notation:

$$
R_{i}^{(m n)}=x_{m i}-x_{n i},
$$




$$
R^{(m n)}=\left[\sum_{i=1}^{3}\left(x_{m i}-x_{n i}\right)^{2}\right]^{\frac{1}{2}}
$$

The potential energy of the ions is

$$
V=\frac{M}{2} \sum_{n=1}^{N} \sum_{i=1}^{3} \omega_{i}^{2} x_{n i}^{2}+\frac{Z^{2} e^{2}}{8 \pi \epsilon_{0}} \sum_{\substack{n, m=1 \\ m \neq n}}^{N} \frac{1}{R^{(m n)}} .
$$

Thus we have:

$$
\begin{aligned}
\frac{\partial V}{\partial x_{m i}}= & M \omega_{i}^{2}\left(x_{m i}-\frac{\ell^{3} \omega_{3}^{2}}{\omega_{i}^{2}} \sum_{\substack{p=1 \\
p \neq m}}^{N} \frac{R_{i}^{(m p)}}{R^{(m p)^{3}}}\right), \\
\frac{\partial^{2} V}{\partial x_{m i} \partial x_{n j}}= & M \omega_{i}^{2}\left[\delta_{m n} \delta_{i j}-\frac{\ell^{3} \omega_{3}^{2}}{\omega_{i}^{2}} \sum_{\substack{p=1 \\
p \neq m}}^{N} \frac{\delta_{m n}-\delta_{p n}}{R^{(m p)^{3}}}\left(\delta_{i j}-3 \frac{R_{i}^{(m p)} R_{j}^{(m p)}}{R^{(m p)^{2}}}\right)\right], \\
\frac{\partial^{3} V}{\partial x_{m i} \partial x_{n j} \partial x_{p k}}= & 3 M \ell^{3} \omega_{3}^{2} \sum_{\substack{q=1 \\
q \neq m}}^{N}\left(\delta_{m n}-\delta_{q n}\right)\left(\delta_{m p}-\delta_{q p}\right) \\
& \times\left(\frac{\delta_{i j} R_{k}^{(m q)}+\delta_{i k} R_{j}^{(m q)}+\delta_{j k} R_{i}^{(m q)}}{R^{(m q)^{5}}}-5 \frac{R_{i}^{(m q)} R_{j}^{(m q)} R_{k}^{(m q)}}{R^{(m q)^{7}}}\right)
\end{aligned}
$$

These terms can be evaluated at equilibrium by making the following substitutions:

$$
\begin{aligned}
\bar{R}_{i}^{(m n)} & =\ell \delta_{i 3}\left(u_{m}-u_{n}\right), \\
\bar{R}^{(m n)} & =\ell\left|u_{m}-u_{n}\right| .
\end{aligned}
$$

We thus obtain:

$$
\begin{aligned}
\left.\frac{\partial V}{\partial x_{m i}}\right|_{0} & =M \omega_{i}^{2} \delta_{i 3}\left(u_{m}-\sum_{\substack{n=1 \\
n \neq m}}^{N} \frac{\operatorname{sgn}\left(u_{m}-u_{n}\right)}{\left(u_{m}-u_{n}\right)^{2}}\right), \\
\left.\frac{\partial^{2} V}{\partial x_{m i} \partial x_{n j}}\right|_{0} & =M \omega_{3}^{2}\left[\delta_{m n} \delta_{i j} \frac{\omega_{i}^{2}}{\omega_{3}^{2}}-\sum_{\substack{p=1 \\
p \neq m}}^{N} \frac{\delta_{m n}-\delta_{p n}}{\left|u_{m}-u_{p}\right|^{3}}\left(\delta_{i j}-3 \delta_{i 3} \delta_{j 3}\right)\right],
\end{aligned}
$$




$$
\begin{aligned}
\left.\frac{\partial^{3} V}{\partial x_{m i} \partial x_{n j} \partial x_{p k}}\right|_{0}= & \frac{3 M \omega_{3}^{2}}{\ell} \sum_{\substack{q=1 \\
q \neq m}}^{N} \frac{\operatorname{sgn}\left(u_{m}-u_{q}\right)\left(\delta_{m n}-\delta_{q n}\right)\left(\delta_{m p}-\delta_{q p}\right)}{\left(u_{m}-u_{q}\right)^{4}} \\
& \times\left(\delta_{i j} \delta_{k 3}+\delta_{i k} \delta_{j 3}+\delta_{j k} \delta_{i 3}-5 \delta_{i 3} \delta_{j 3} \delta_{k 3}\right) .
\end{aligned}
$$

Equation (A.9) give the equations that determine the equilibrium positions $u_{m}$. From eq.(A.10) we can write

$\left.\frac{1}{2} \sum_{m, n=1}^{N} \sum_{i, j=1}^{3} \frac{\partial^{2} V}{\partial x_{m i} \partial x_{n j}}\right|_{0} \xi_{m i} \xi_{n j}=\frac{M \omega_{3}^{2}}{2} \sum_{m, n=1}^{N} A_{m n} \xi_{m 3} \xi_{n 3}+B_{m n}\left(\xi_{m 1} \xi_{n 1}+\xi_{m 2} \xi_{n 2}\right)$,

with

$$
A_{m n}=\delta_{m n}+2 \sum_{\substack{p=1 \\ p \neq m}}^{N} \frac{\delta_{m n}-\delta_{p n}}{\left|u_{m}-u_{p}\right|^{3}}
$$

and

$$
B_{m n}=\frac{\delta_{m n}}{\alpha}-\sum_{\substack{p=1 \\ p \neq m}}^{N} \frac{\delta_{m n}-\delta_{p n}}{\left|u_{m}-u_{p}\right|^{3}}
$$

which are equivalent to the expressions given in Section 2. And, finally:

$$
\begin{aligned}
\frac{1}{6} & \left.\sum_{m, n, p=1}^{N} \sum_{i, j, k=1}^{3} \frac{\partial^{3} V}{\partial x_{m i} \partial x_{n j} \partial x_{p k}}\right|_{0} \xi_{m i} \xi_{n j} \xi_{p k} \\
= & \frac{M \omega_{3}^{2}}{2 \ell} \sum_{m n p=1}^{N} C_{m n p} \xi_{p 3}\left(2 \xi_{m 3} \xi_{n 3}-3 \xi_{m 1} \xi_{n 1}-3 \xi_{m 2} \xi_{n 2}\right),
\end{aligned}
$$

where

$$
C_{m n p}=\sum_{\substack{q=1 \\ q \neq m}}^{N} \frac{\operatorname{sgn}\left(u_{q}-u_{m}\right)\left(\delta_{m n}-\delta_{q n}\right)\left(\delta_{m p}-\delta_{q p}\right)}{\left(u_{m}-u_{q}\right)^{4}} .
$$

\section{Appendix B}

The following tables list the non-zero values of the mode cross-coupling coefficients $D_{m n p}$ defined by eq.(2.20) for crystals of 2 to 10 ions, together with the associated resonant anisotropy parameter $\alpha$. Other values may be determined via the symmetry relation $D_{m n p}=D_{m p n}=D_{n p m}=D_{n m p}=D_{p m n}=D_{p n m}$. Note however that the value of $\alpha$ is symmetric only under interchange of the first two indices. 
Table 2a: Coupling constants for resonances of the first kind (in which a transverse phonon creates a longitudinal phonon and a transverse phonon).

\begin{tabular}{|c|c|c|c|c|c|c|c|c|}
\hline$\{\mathrm{m}, \mathrm{n}, \mathrm{p}\}$ & $D_{m n p}$ & $\alpha_{\text {res }}$ & $\{\mathrm{m}, \mathrm{n}, \mathrm{p}\}$ & $D_{m n p}$ & $\alpha_{\text {res }}$ & $\{\mathrm{m}, \mathrm{n}, \mathrm{p}\}$ & $D_{m n p}$ & $\alpha_{\text {res }}$ \\
\hline $\mathrm{N}=6$ & & & $\mathrm{~N}=7$ & & & & & \\
\hline$\{3,6,3\}$ & $2.8395 \mathrm{e}-4$ & 0.11575 & $\{4,7,3\}$ & $5.4794 \mathrm{e}-4$ & 0.087700 & & & \\
\hline \multicolumn{9}{|l|}{$\mathrm{N}=8$} \\
\hline$\{3,8,3\}$ & $3.1152 \mathrm{e}-06$ & 0.062943 & $\{4,8,4\}$ & $1.0031 \mathrm{e}-3$ & 0.069797 & $\{5,8,3\}$ & $7.4469 \mathrm{e}-4$ & 0.068879 \\
\hline \multicolumn{9}{|l|}{$\mathrm{N}=9$} \\
\hline$\{3,9,4\}$ & $5.7509 \mathrm{e}-06$ & 0.054162 & $\{4,9,3\}$ & $5.7509 \mathrm{e}-06$ & 0.049960 & $\{5,9,4\}$ & $1.2967 \mathrm{e}-3$ & 0.056583 \\
\hline$\{6,9,3\}$ & $8.8502 \mathrm{e}-4$ & 0.055637 & & & & & & \\
\hline \multicolumn{9}{|l|}{$\mathrm{N}=10$} \\
\hline$\{3,10,3\}$ & $5.9416 \mathrm{e}-08$ & 0.035465 & $\{3,10,5\}$ & $7.3591 \mathrm{e}-06$ & 0.046313 & $\{4,10,4\}$ & $1.0248 \mathrm{e}-05$ & 0.044211 \\
\hline$\{5,10,3\}$ & $7.3591 \mathrm{e}-06$ & 0.040715 & $\{5,10,5\}$ & $1.5984 \mathrm{e}-3$ & 0.047291 & $\{6,10,4\}$ & $1.4703 \mathrm{e}-3$ & 0.046822 \\
\hline$\{7,10,3\}$ & $9.8451 \mathrm{e}-4$ & 0.045964 & & & & & & \\
\hline
\end{tabular}


Table 2b: Resonances of the second kind, (in which a longitudinal phonon creates two transverse phonons).

\begin{tabular}{|c|c|c|c|c|c|c|c|c|}
\hline$\{\mathrm{m}, \mathrm{n}, \mathrm{p}\}$ & $D_{m n p}$ & $\alpha_{\text {res }}$ & $\{\mathrm{m}, \mathrm{n}, \mathrm{p}\}$ & $D_{m n p}$ & $\alpha_{\text {res }}$ & $\{\mathrm{m}, \mathrm{n}, \mathrm{p}\}$ & $D_{m n p}$ & $\alpha_{\text {res }}$ \\
\hline $\mathrm{N}=2$ & & & $\mathrm{~N}=3$ & & & & & \\
\hline$\{2,2,2\}$ & -1.1225 & 0.57143 & $\{3,2,3\}$ & -1.5754 & 0.30917 & $\{3,3,2\}$ & -1.5754 & 0.31746 \\
\hline \multicolumn{9}{|l|}{$\mathrm{N}=4$} \\
\hline$\{3,3,4\}$ & -1.8332 & 0.21132 & $\{4,2,4\}$ & -1.9493 & 0.19337 & \multirow[t]{2}{*}{$4,3,3$} & \multirow[t]{2}{*}{-1.8332} & \multirow[t]{2}{*}{0.20560} \\
\hline$\{4,4,2\}$ & -1.9493 & 0.20391 & $\{4,4,4\}$ & 2.1084 & 0.15429 & & & \\
\hline \multicolumn{9}{|l|}{$\mathrm{N}=5$} \\
\hline$\{4,3,5\}$ & -1.9683 & 0.14895 & $\{4,4,4\}$ & -0.89355 & 0.15387 & \multirow{3}{*}{$\begin{array}{l}\{5,2,5\} \\
\{5,4,5\}\end{array}$} & \multirow{3}{*}{$\begin{array}{l}-2.2887 \\
3.1611\end{array}$} & \multirow{3}{*}{$\begin{array}{l}0.13340 \\
0.11561\end{array}$} \\
\hline$\{5,3,4\}$ & -1.9683 & 0.14187 & $\{5,4,3\}$ & -1.9683 & 0.14619 & & & \\
\hline$\{5,5,2\}$ & -2.2887 & 0.14311 & $\{5,5,4\}$ & 3.1611 & 0.11668 & & & \\
\hline \multicolumn{9}{|l|}{$\mathrm{N}=6$} \\
\hline$\{4,4,6\}$ & -1.8850 & 0.11439 & $\{5,3,6\}$ & -2.0625 & 0.10985 & $\{5,4,5\}$ & $8.1287 \mathrm{e}-2$ & 0.11527 \\
\hline$\{5,5,6\}$ & 4.2528 & 0.092393 & $\{6,2,6\}$ & -2.6084 & 0.098229 & $\{6,3,5\}$ & -2.0625 & 0.10398 \\
\hline$\{6,4,4\}$ & -1.8850 & 0.10784 & $\{6,4,6\}$ & 4.0185 & 0.088947 & $\{6,5,3\}$ & -2.0625 & 0.10937 \\
\hline$\{6,5,5\}$ & 4.2528 & 0.091510 & $\{6,6,2\}$ & -2.6084 & 0.10658 & $\{6,6,4\}$ & 4.0185 & 0.091151 \\
\hline$\{6,6,6\}$ & -2.3706 & 0.075762 & & & & & & \\
\hline \multicolumn{9}{|l|}{$\mathrm{N}=7$} \\
\hline$\{6,3,7\}$ & -2.1382 & 0.084291 & $\{6,5,7\}$ & 4.9336 & 0.074398 & $\{6,6,6\}$ & 4.3077 & 0.075526 \\
\hline$\{7,2,7\}$ & -2.9149 & 0.075728 & $\{7,3,4\}$ & $5.4794 \mathrm{e}-4$ & 0.088234 & $\{7,3,6\}$ & -2.1382 & 0.079773 \\
\hline$\{7,4,5\}$ & -1.7957 & 0.082749 & $\{7,4,7\}$ & 4.7748 & 0.070376 & $\{7,5,4\}$ & -1.7957 & 0.084569 \\
\hline$\{7,5,6\}$ & 4.9336 & 0.072836 & $\{7,6,3\}$ & -2.1382 & 0.085059 & $\{7,6,5\}$ & 4.9336 & 0.074000 \\
\hline$\{7,6,7\}$ & -3.8751 & 0.062565 & $\{7,7,2\}$ & -2.9149 & 0.082796 & $\{7,7,4\}$ & 4.7748 & 0.073152 \\
\hline$\{7,7,6\}$ & -3.8751 & 0.062861 & & & & & & \\
\hline \multicolumn{9}{|l|}{$\mathrm{N}=8$} \\
\hline$\{6,6,8\}$ & 5.2871 & 0.062147 & $\{7,3,8\}$ & -2.2035 & 0.066792 & $\{7,5,8\}$ & 5.4173 & 0.060792 \\
\hline$\{7,6,7\}$ & 3.6190 & 0.062378 & $\{7,7,6\}$ & 3.6190 & 0.062674 & $\{7,7,8\}$ & -5.8640 & 0.053277 \\
\hline$\{8,2,8\}$ & -3.2119 & 0.060393 & $\{8,3,5\}$ & $7.4469 \mathrm{e}-4$ & 0.069615 & $\{8,3,7\}$ & -2.2035 & 0.063341 \\
\hline$\{8,4,6\}$ & -1.7158 & 0.065623 & $\{8,4,8\}$ & 5.4691 & 0.057068 & $\{8,5,5\}$ & -1.5767 & 0.067200 \\
\hline$\{8,5,7\}$ & 5.4173 & 0.059134 & $\{8,6,4\}$ & -1.7158 & 0.068081 & $\{8,6,6\}$ & 5.2871 & 0.060526 \\
\hline$\{8,6,8\}$ & -5.1946 & 0.052159 & $\{8,7,3\}$ & -2.2035 & 0.068170 & $\{8,7,5\}$ & 5.4173 & 0.061000 \\
\hline$\{8,7,7\}$ & -5.8640 & 0.053018 & $\{8,8,2\}$ & -3.2119 & 0.066389 & $\{8,8,4\}$ & 5.4691 & 0.060029 \\
\hline$\{8,8,6\}$ & -5.1946 & 0.052896 & $\{8,8,8\}$ & 2.1526 & 0.046043 & & & \\
\hline \multicolumn{9}{|l|}{$\mathrm{N}=9$} \\
\hline$\{7,6,9\}$ & 5.4146 & 0.052246 & $\{7,7,8\}$ & 1.6006 & 0.053125 & $\{8,3,9\}$ & -2.2623 & 0.054311 \\
\hline$\{8,5,9\}$ & 5.7898 & 0.050462 & $\{8,6,8\}$ & 2.6715 & 0.052000 & $\{8,7,7\}$ & 1.6006 & 0.052867 \\
\hline$\{8,7,9\}$ & -7.3423 & 0.045500 & $\{8,8,6\}$ & 2.6715 & 0.052744 & $\{8,8,8\}$ & -7.5531 & 0.045900 \\
\hline$\{9,2,9\}$ & -3.5017 & 0.049433 & $\{9,3,6\}$ & $8.8502 \mathrm{e}-4$ & 0.056425 & $\{9,3,8\}$ & -2.2623 & 0.051650 \\
\hline$\{9,4,5\}$ & $1.2967 \mathrm{e}-3$ & 0.056883 & $\{9,4,7\}$ & -1.6468 & 0.053425 & $\{9,4,9\}$ & 6.1214 & 0.047245 \\
\hline$\{9,5,6\}$ & -1.4030 & 0.054734 & $\{9,5,8\}$ & 5.7898 & 0.048927 & $\{9,6,5\}$ & -1.4030 & 0.055586 \\
\hline$\{9,6,9\}$ & -6.3927 & 0.044000 & $\{9,7,4\}$ & -1.6468 & 0.056011 & $\{9,7,6\}$ & 5.4146 & 0.050996 \\
\hline$\{9,7,8\}$ & -7.3423 & 0.044970 & $\{9,8,3\}$ & -2.2623 & 0.055954 & $\{9,8,5\}$ & 5.7898 & 0.051126 \\
\hline$\{9,8,7\}$ & -7.3423 & 0.045400 & $\{9,8,9\}$ & 3.8083 & 0.039874 & $\{9,9,2\}$ & -3.5017 & 0.054558 \\
\hline$\{9,9,4\}$ & 6.1214 & 0.050180 & $\{9,9,6\}$ & -6.3927 & 0.045081 & $\{9,9,8\}$ & 3.8083 & 0.039988 \\
\hline \multicolumn{9}{|l|}{$\mathrm{N}=10$} \\
\hline$\{7,7,10\}$ & 5.2107 & 0.044976 & $\{8,6,10\}$ & 5.4385 & 0.044332 & $\{8,7,9\}$ & -0.42626 & 0.045380 \\
\hline$\{8,8,8\}$ & -1.9896 & 0.045779 & $\{8,8,10\}$ & -8.6478 & 0.039673 & $\{9,3,10\}$ & -2.3165 & 0.045099 \\
\hline$\{9,4,9\}$ & 3.3150 & 0.047113 & $\{9,5,10\}$ & 6.0930 & 0.042512 & $\{9,6,9\}$ & 1.6336 & 0.043878 \\
\hline$\{9,7,8\}$ & -0.42626 & 0.044846 & $\{9,7,10\}$ & -8.5012 & 0.039123 & $\{9,8,7\}$ & -0.42626 & 0.045279 \\
\hline$\{9,8,9\}$ & -8.0166 & 0.039760 & $\{9,9,6\}$ & 1.6336 & 0.044957 & $\{9,9,8\}$ & -8.0166 & 0.039874 \\
\hline$\{9,9,10\}$ & 6.3437 & 0.035161 & $\{10,2,10\}$ & -3.7855 & 0.041300 & $\{10,3,7\}$ & 9.8451 e-04 & 0.046733 \\
\hline$\{10,3,9\}$ & -2.3165 & 0.043017 & $\{10,4,6\}$ & $1.4703 \mathrm{e}-3$ & 0.047276 & $\{10,4,8\}$ & -1.5874 & 0.044422 \\
\hline$\{10,4,10\}$ & 6.7435 & 0.039798 & $\{10,5,7\}$ & -1.2642 & 0.045500 & $\{10,5,7\}$ & -1.2642 & 0.045500 \\
\hline$\{10,5,9\}$ & 6.0930 & 0.041163 & $\{10,6,6\}$ & -1.1721 & 0.046259 & $\{10,6,8\}$ & 5.4385 & 0.042265 \\
\hline$\{10,6,10\}$ & -7.5034 & 0.037564 & $\{10,7,5\}$ & -1.2642 & 0.046721 & $\{10,7,7\}$ & 5.2107 & 0.043067 \\
\hline$\{10,7,9\}$ & -8.5012 & 0.038483 & $\{10,8,4\}$ & -1.5874 & 0.046923 & $\{10,8,6\}$ & 5.4385 & 0.043500 \\
\hline$\{10,8,8\}$ & -8.6478 & 0.039085 & $\{10,8,10\}$ & 5.3952 & 0.034687 & $\{10,9,3\}$ & -2.3165 & 0.046823 \\
\hline$\{10,9,5\}$ & 6.0930 & 0.043468 & $\{10,9,7\}$ & -8.5012 & 0.039269 & $\{10,9,9\}$ & 6.3437 & 0.035057 \\
\hline$\{10,10,2\}$ & -3.7855 & 0.045724 & $\{10,10,4\}$ & 6.7435 & 0.042604 & $\{10,10,6\}$ & -7.5034 & 0.038860 \\
\hline$\{10,10,8\}$ & 5.3952 & 0.035000 & $\{10,10,10\}$ & -1.7362 & 0.031318 & & & \\
\hline
\end{tabular}




\section{References}

[1] J. I. Cirac and P. Zoller: Phys. Rev. Lett. 74, 4094 (1995).

[2] C. Monroe, D. M. Meekhof, B. E. King, W. M. Itano and D. J. Wineland: Phys. Rev. Lett. 75, 4714 (1995).

[3] M. A. Nielsen and I. L. Chuang: Quantum Computation and Quantum Information, (Cambridge University Press, Cambridge, 2000), section 7.6 .

[4] D. J. Wineland, C. Monroe, W. M. Itano, D. Leibfried, B. King, and D. M. Meekhof: J. Res. Natl. Inst. Stand. Technol. 103, 259 (1998).

[5] D. F. V. James: Fort. d. Phys. 48, 823(2000).

[6] D. Kielpinski, C. Monroe and D. J. Wineland: Nature 417, 709 (2002).

[7] M. Šašura and V. Bužek: J. Mod. Opt. 49, 1593 (2002).

[8] C. A. Sackett, D. Kielpinski, B. E. King, C. Langer, V. Meyer, C. J. Myatt, M. Rowe, Q. A. Turchette, W. M. Itano, D. J. Wineland and C. Monroe: Nature 404256 (2000).

[9] M. A. Rowe, D. Kielpinski, V. Meyer, C. A. Sackett, W. M. Itano, C. Monroe and D. J. Wineland: Nature 409791 (2001).

[10] J. Eschner, C. Raab, F. Schmidt-Kaler and R. Blatt: Nature 413495 (2001).

[11] S. Earnshaw: Trans. Cambridge Phil. Soc. 7, 97 (1842). See also, for instance, J. A. Stratton: Electromagnetic Theory (McGraw-Hill, New York, 1941), p. 116.

[12] P. K. Ghosh: Ion Traps (Clarendon Press, Oxford, 1995).

[13] D. J. Berkeland, J. D. Miller, J. C. Bergquist, W. M. Itano and D. J. Wineland: Phys. Rev. Lett. 802089 (1998).

[14] E. Fermi, J. Pasta and S. Ulam: "Studies of Nonlinear Problems I," Los Alamos Scientific Laboratory report \# LA-1940 (May, 1955); reprinted in S. Ulam: Stanilaw Ulam: Sets, Numbers and Universes; Selected Works (W. A. Beyer, J. Mycielski and G.-C. Rota, eds.; MIT Press, Cambridge MA, 1974), p.490.

[15] See, for example, N. Gisin, J. G. Rarity and G. Weihs in The Physics of Quantum Information (D. Bouwmeester, A. Ekert, A. Zeilinger, eds.; Springer, Berlin, 2000), section 3.4, p.53. 
[16] D. J. Berkeland, J. D. Miller, J. C. Berquist, W. M. Itano and D. J. Wineland: J. Appl. Phys. 835025 (1998).

[17] A. M. Steane: Appl. Phys. B 64, 623 (1997).

[18] D. F. V. James: Appl. Phys. B 66, 181 (1998).

[19] T. P. Meyrath and D. F. V. James: Phys. Lett. A 240, 37 (1998).

[20] D. Kielpinski, B. E. King, C. J. Myatt, C. A. Sackett, Q. A. Turchette, W. M. Itano, C. Monroe, D. J. Wineland and W. H. Zurek: Phys. Rev. A 61, 032310 (2000).

[21] G. Morigi and H. Walther: Eur. Phys. J. D 13, 261 (2000); erratum: ibid. 15, 137 (2000).

[22] H. C. Nagerl, D. Leibfried, F. Schmidt-Kaler, J. Eschner and R. Blatt: Optics Express 3, 89 (1998).

[23] D. Enzer, M. M. Schauer, J. J. Gomez, M. S. Gulley, M. H. Holzscheiter, P. G. Kwiat, S. K. Lamoreaux, C. G. Peterson, V. D. Sandberg, D. Tupa, A. G. White, R. J. Hughes and D. F. V. James: Phys. Rev. Lett. 85, $2466(2000)$.

[24] X. Y. Chu, M. Holzki, R. Alheit and G. Werth: Int. J. Mass. Spectrom. Ion Processes 173, 107 (1998). Note that these authors use the word "mode" in a different context to that used in this paper.

[25] Q. A. Turchette, D. Kielpinski, B. E. King, D. Leibfried, D. M. Meekhof, C. J. Myatt, M. A. Rowe, C. A. . Sackett, C. S. Wood, W. M. Itano, C. Monroe, and D. J. Wineland: Phys. Rev. A 61, 063418 (2000).

[26] L. I. Schiff, Quantum Mechanics (McGraw-Hill, New York, 2nd edition 1968), section 35.

[27] A. G. White, P. G. Kwiat, D. F. V. James and P. H. Eberhard: Phys. Rev. Lett. 83, 3103 (1999).

[28] P. G. Kwiat: J. Mod. Opt. 442173 (1997). 